\title{
INTERACTIVE TOOL FOR MOBILE ROBOT MOTION PLANNING
}

\author{
J.L. Guzmán*a, M. Berenguel ${ }^{\text {a }}$, F. Rodríguez ${ }^{\text {a }}$, S. Dormidob \\ ${ }^{a}$ Dep. Lenguajes y Computación, Universidad de Almería, Ctra. Sacramento $s / n$ \\ 04120 Almería, Spain. Tlf: + 34 950015849. Fax: + 34950015129. \\ E-mail:joguzman@ual.es \\ ${ }^{\mathrm{b}}$ Dep. Informática y Automática, ETSI Informática, UNED, C. Juan del Rosal, 16 \\ 28040 Madrid, Spain.
}

\begin{abstract}
Mobile robotics is a subject included in several engineering curricula at present. The continuous increase in computer processing power allows the use of interactive tools to merge the synthesis and analysis phases of classical engineering iterative design techniques into one, being possible to apply these advantages to the robotics field. An interactive tool is presented in this work aimed at facilitating the understanding of several well-known algorithms and techniques involved in solving mobile robot motion problems, from those modelling the mechanics of mobility to those used in navigation. The paper also discuses how the tool can be used in an introductory course of mobile robotics.
\end{abstract}

Key words: mobile robotics, interactivity, simulation, kinematics, robot motion

\section{Introduction}

This paper presents an interactive tool for understanding basic concepts involved in mobile robotics, and also discusses how it can be used in an introductory course. Interactive tools are becoming key elements in different engineering courses and mobile robotics is an educational field where the advantages of this learning paradigm can be better exploited. The theory can be learned through textbooks, inspiring lectures, and active study. The ability to solve practical problems relies on good skill in using theory and in breaking down large problems into manageable subproblems. One of the important tasks for teachers in engineering is to transmit to the students not only the formal and logic structure of the discipline, but also, and certainly with much more emphasis, the strategic and intuitive aspects of the subject [15]. These 
last aspects are probably much more difficult to make explicit and assimilate for students, precisely because they lie very often in the less conscious substrata of the expert's activity. Interactive tools are considered a great stimulus for developing the student's intuition and they attempt to demystify abstract mathematical concepts through visualization for specifically chosen examples [14]. Although the term Interactivity can be defined in several ways [14], from a teaching point of view an Interactive Tool can be defined as a collection of graphical windows whose components are active, dynamic, and/or clickable; and that is intended to explain just a few concepts. The use of interactive and instructional graphic tools would reinforce active participation of students. For educators, this kind of tools can provide a very useful way to test main ideas and to realize how difficult explaining a particular concept to students is $[14,31]$.

Mobile robotics is a relatively new research area that deals with the control of autonomous and semiatonomus vehicles. It is the domain where literally the "rubber meets the road" for many algorithms in path planning, knowledge representation, sensing and reasoning. An autonomous mobile robot is a machine able to extract information from its environment and use knowledge about its world to move safely in a meaningful and purposive manner, it can operate on its own without a human directly controlling it [8]. The primary task of a mobile robot is environmental navigation as basis for more useful tasks.

In other words, mobile robotics deals with the problem of moving a mobile robot in any environment following a free collision path. It involves many tasks related to algorithms for path planning and tracking, knowledge representation, sensing, and reasoning. For this reason, mobile robotics combines many disciplines, including electrical engineering, automatic control, computer science, mechanical engineering, artificial intelligent and others [2]. Mobile robotics can be understood as a navigation problem, described as the action of directing a mobile robot to traverse a specific environment. The main aim in any navigation scheme is to reach a destination without moving away from the goal or crashing with objects [25], [2]. Navigation is divided into three tasks: mapping, planning and tracking [17], [25]. If the map of the environment is considered as an input to the system (the mapping problem has been solved), and under a typical 2D navigation assumption [17], [25], [2] the navigation process involves two kinds of problems:

- Path planning. It is a geometric problem related with the robot dimensions and the obstacles located in the navigation space. Its solution provides a sequence of positions that joins the initial and goal points defining the free trajectory (without obstacles) to be followed by the robot.

- Path tracking. It is a control problem related with the constraints on the robot movements, actuators, and the time elapsed in the navigation. Its so- 
lution provides, all the time, the set point to each actuator aimed at reaching or following (sometimes in a prescribed time) the trajectory obtained when solving the geometric problem.

In this work, a typical basic course of mobile robot motion planning has been followed, as that described in [2] which is used in the Massachusetts Institute of Technology (MIT) and other prestigious universities listed in http://wwwcvr.ai.uiuc.edu/ $\sim$ seth/RMPbook/. The aim of the interactive tool presented in this paper is to help studying the classical notion paradigms, basically focused on[2]:

- Roadmap algorithms, like Visibility graph and Voronoi Diagram.

- Potential algorithms like Potential Functions and Wave front.

- Bug algorithms.

- Cellular decomposition.

Evidently, there exist other algorithms within the previous paradigms not included in the tool, such as the Probabilistic Methods (Probabilistic Roadmap Planning, Expansive-Spaces Tree Planning or Probabilistic Roadmap of Tree, etc.) [2], which are susceptible to be included in the tool in the future.

The solution of the path planning task depends on the knowledge the robot has of the navigation space, so the previous algorithms can be grouped considering this idea. Basically, global algorithms (such as Visibility Graph, Voronoi Diagram, Cell decomposition, and Wave front) will be used if the dimensions and locations of all the obstacles are well-known (deliberative navigation). If this information is unknown, the use of local algorithms (such as Bug and Potential function algorithms) is required (reactive navigation). With both global and local algorithms it is necessary to know or estimate the robot position at each time. In real applications different techniques and/or sensors are used to obtain the actual robot position (odometry, inertial methods, GPS, etc.). The integration of the robot kinematics or dynamics provides an approximation to the real position that can be used for simulation purposes, as done in this application.

Therefore, mobile robotics involves many disciplines and the navigation problem requires several steps that must be well studied and understood by engineering students. Based on the authors' experience, teaching and learning of the involved concepts could be addressed using the new interactive methodologies developed during the last years and having a great impact in the field of Automatic Control $[14,18,35,4,11,7]$. As commented in [7], the continuous increase in computer processing power allows the use of interactive tools to merge the synthesis and analysis phases of classical engineering iterative design techniques into one, in such a way that the modification of any configuration, parameter, or element in the system produces an immediate effect in the rest 
and thus the design procedure becomes really dynamic, allowing to identify much more easily the compromises that can be achieved. So, the main contribution of this paper is to present an interactive tool that helps teaching and learning main concepts and techniques involved in mobile robotics by simulation over a 2D environment defined by the Cartesian coordinates $(x, y)$. This tool is being used in an introductory course of mobile robotics for Computer Science engineering students.

\section{Related work}

The vertiginous advances that undergone the new teaching technologies have had a great impact in the scope of robotics, mainly in the field of robot manipulators. During the last years, multiple tools, virtual, and remote labs have been developed to allow the students work and learn at home or through the WWW from any part of the world without temporal constraints $[5,3,33,32]$ and providing interactive learning [19]. Several teaching tools have also been published in the field of mobile robotics (e.g. [10,1,4]), but with less impact than those developed in the scope of robot manipulators.

In [9] an interactive autonomous robot motion planning system developed in Java is described. This interactive system enables users to set up the working environment by creating obstacles and a robot of different shapes, specifying starting and goal positions and setting other path or environment parameters from a user-friendly interface. The main disadvantage of this tool is that only deliberative navigation is considered where the Minkowski sum and Visibility Graph are the unique algorithms implemented. Furthermore, robot kinematics are not used. On the other hand, an interactive system based on Virtual Reality where the aim consists on reconstructing the real environment based on the real sensor is presented in [27]. A simulation option is also included where the robot is based on tricycle kinematics, the path planning is defined by the user where the operator enters several possible points to track, and the minimum path is obtained using Dijkstra's algorithm [6].

Another tools are AMORsim [28], Khepera simulator [24], Stage [34], SIMROBOT [16], and HEMERO [23] which are mainly focused on particular kinematic study avoiding path planing problems. For instance, HEMERO is a toolbox for the study of manipulator and mobile robots. The tool combines Matlab and Simulink for the representation the kinematic and dynamic modelling of the robots. The numerical results are illustrated by means of static simple graphics generated with Matlab without interactive behavior. The tool presented in this paper has been oriented to cover the main features of the previous tools in such a way that the different aspects considered in a basic course of mobile robotics are included. Its development was motivated twofold. 
It can help the lecturer to show how mobile robots behave depending of the selected kinematics, path planning algorithms, and robot control algorithms. In addition, this tool can help the students to put into practice and understand themselves the learned concepts, and to analyze how algorithms behave previous to use them in real robots.

The tool presented in this work facilitates the understanding of the algorithms used in solving mobile robotics problems. In the first version [13] the tool had the possibility of working with known environments and robots with differential mechanism, where the navigation from the initial to the goal point was performed using a visibility graph algorithm for path planning and the pure pursuit method for path tracking (similar to the tool presented in [10]). The objective of the current version of the tool is to extend its capabilities in some aspects. In the case of navigation algorithms, both global algorithms (using global or vertex maps - Visibility graphs, free-space maps - Voronoi diagrams, composite-space maps - Quadtrees, propagation-space maps - WaveFront) and local algorithms (potential, BUG and VISBUG) have been considered.

Three different robot kinematics have also been included: differential mechanism, tricycle/Ackermann, and synchronous. Many different parameters can be selected and/or modified using the tool user interface, such as those associated to the navigation algorithm, the initial and goal positions of the mobile robot, the robot kinematics and associated dimensions, the number, morphology and position of different objects in the environment, etc. The user can interactively visualize the effect that these modifications have on the robot trajectory to navigate from the initial to the goal position (the results can be shown in one run or using a step by step procedure). The selection of the features included in the tool has been based on the typical contents of introductory courses of mobile robotics.

\section{Description of the tool}

This section describes the main features of the tool, programmed with SysQuake, a Matlab-like [22] environment which has strong support for interactive graphics [29]. The interactive tool is free and available for Windows, Mac, and Linux operating systems [12]. Notice that the main feature of the tool - Interactivity - is difficult to be highlighted in a written text. When developing a tool of this kind, the programming of the algorithms is a time consuming aspect, but one of the most important things that the teacher has to have in mind is the organization of the main windows and menus of the tool to facilitate the student the understanding of the theoretical aspects [14]. The main window of the tool is divided into several sections represented in Fig. 1 (basic screen of the tool) which are described in what follows: 


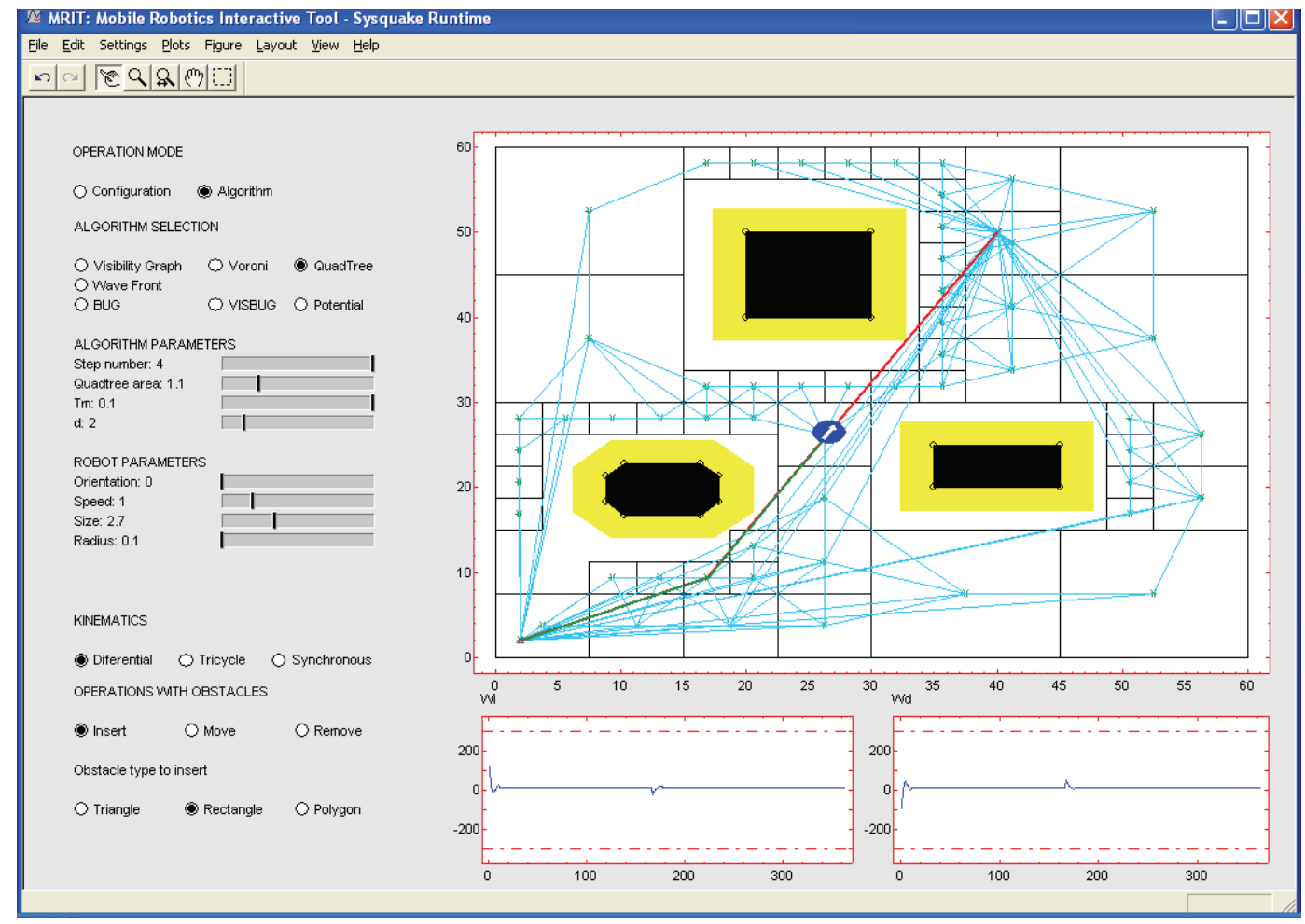

Fig. 1. Main window of the tool showing an example.

- Environment: main window on the right side of the screen. It represents the working space with a set of obstacles where the robot will evolve. It is possible to change the morphology of the obstacles dragging with the mouse over their corners. The original objects are shown in black, their convex envelopes are drawn using dotted lines, and the final object (the convex obstacle enlarged using the robot width) are presented in yellow. Both the origin point (the robot) and the target point that the robot will try to reach can be modified over this graph. The results of the selected planning algorithm are shown on the environment. The shortest path is drawn in red (for the global algorithms), and the path followed by the robot in green colour.

- Operation mode: two operation modes are available in the tool and can be modified using this option. The Configuration mode allows defining the environment, algorithms, and robot related options before running a simulation. The Algorithm mode is used to calculate the minimum-length path (if selected) and run the simulation.

- Algorithm selection and parameters: these two options allow choosing the desired planning algorithm and associated parameters (see Table 1). The parameters of the pure pursuit algorithm implemented for path tracking purposes can also be changed.

- Robot parameters and kinematics: the different initial robot parameters such as size, speed, orientation, and wheel radius can be modified. On the other hand, it is possible to select the robot morphology between differential mech- 
anism, tricycle, and synchronous (see Table 1).

- Obstacle insertion: it allows inserting a new obstacle into the environment, this option being available on the left-bottom corner of the tool. First, the morphology of the obstacle is selected between several options: triangle, rectangle or free polygon. After this selection, the mouse pointer can be placed on the environment location in which the obstacle will be inserted, and then a single click on the left mouse button will perform the action. In the case of the free polygon option, the number of sides must be selected using the slider Sides.

- Control signals: two graphics on the bottom right of the interface (Fig. 1) show the evolution of the control signals of the selected kinematics (the angular velocities of the left and right wheels, $\omega_{i}$ and $\omega_{d}$ in Fig. 1 because the differential kinematic is chosen). Amplitude and/or rate input constraints can be included by dragging in the tool the red vertical lines shown in the graphs.

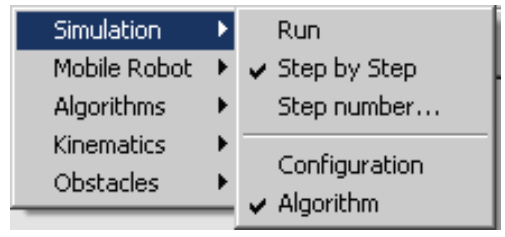

Fig. 2. Settings menu.

- Settings Menu: it is possible to start, pause, and stop the simulation and to show the results step by step. All the options available in the graphical interface can be also modified from this menu (see Fig. 2).

Fig. 3 describes the steps that have to be performed when using the tool. These steps have been explained sequentially but they can be used in any order interactively.

\section{Global and local algorithms}

Global path planning algorithms usually rely on computational geometry methods under several assumptions, such that the space is planar, the robot is the only dynamic object in the navigation space, and its actuators do not present any constraint. These algorithms calculate the optimal trajectory that joins the initial and goal points based on the environment map (dimension and location of the obstacles) ensuring that the robot does not collide with any obstacle. The optimal trajectory can be calculated based on the minimization of the distance between the initial and goal points, the maximization of the distance to the obstacles, the minimum curvature, and other criteria.

The inputs to the local algorithms are the initial and the goal points, and 


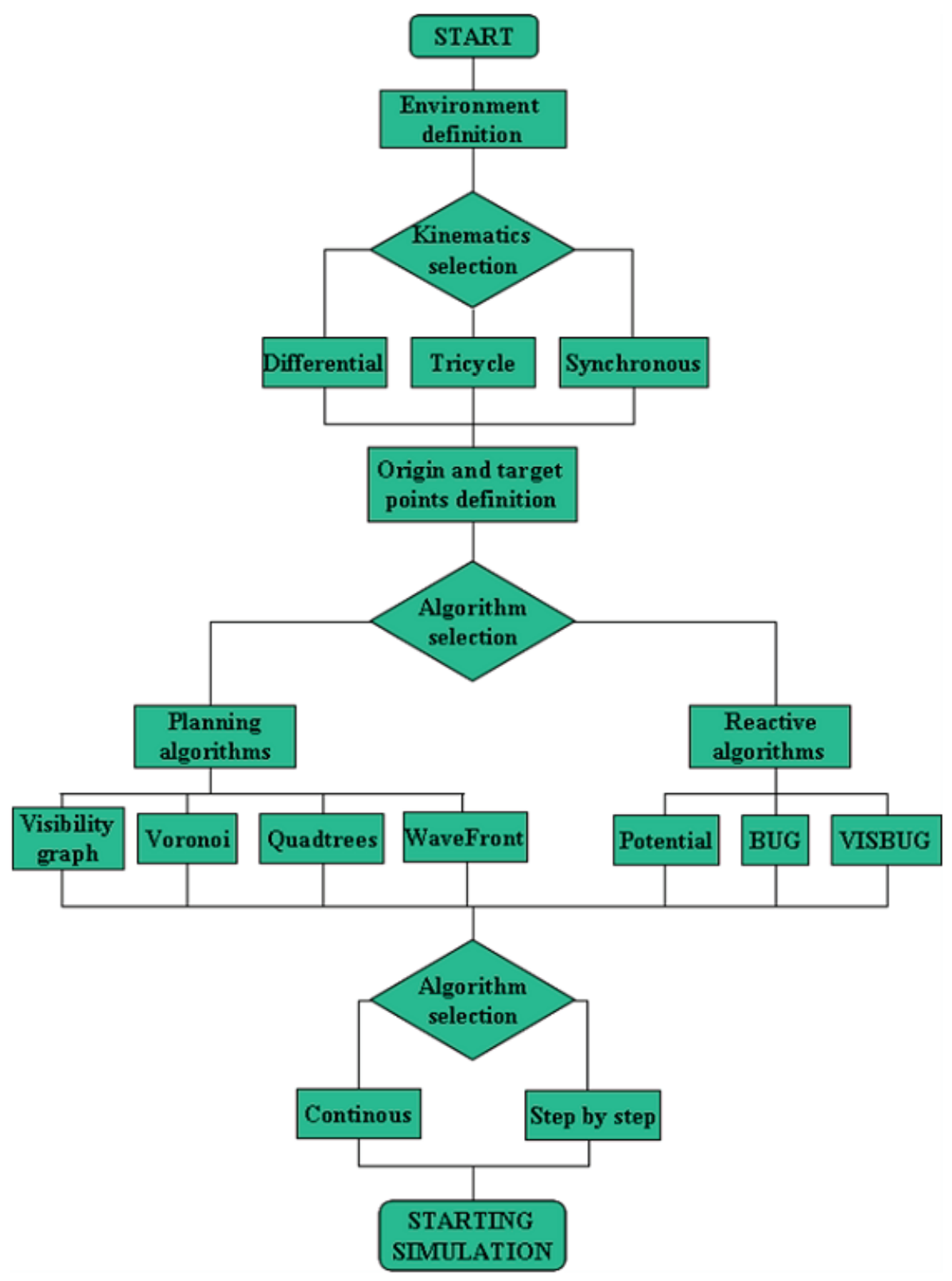

Fig. 3. Steps to be carried out when using the tool.

the information provided by robot sensors (e.g. odometry or distance). They automatically generate the set points to the velocity controllers of the wheel robot to go from the initial point to the goal one avoiding obstacles. The main feature of these algorithms is the necessity of information from the sensorial signals (reactive navigation), since the only hypothesis is that the navigation space is navigable and smooth. 


\subsection{Global algorithms}

The first stage when using global algorithms consists in obtaining all possible collision-free paths, sometimes starting with the calculation of the convex hull for the obstacles, and augmenting the resulting shapes according to the robot dimensions. In order to obtain all possible paths from the initial to the goal points, several algorithms can be used [17], [20], [2], as those implemented in the interactive tool (see Table 1):

- Visibility graph. It is based on the concept of visible points, that are those points (initial, goal, and vertexes of the obstacles) that can be joined each other with a line without crossing any obstacle. In this way, a graph can be generated joining all visible points in the navigation space including the obstacle sides (see Fig. 4).

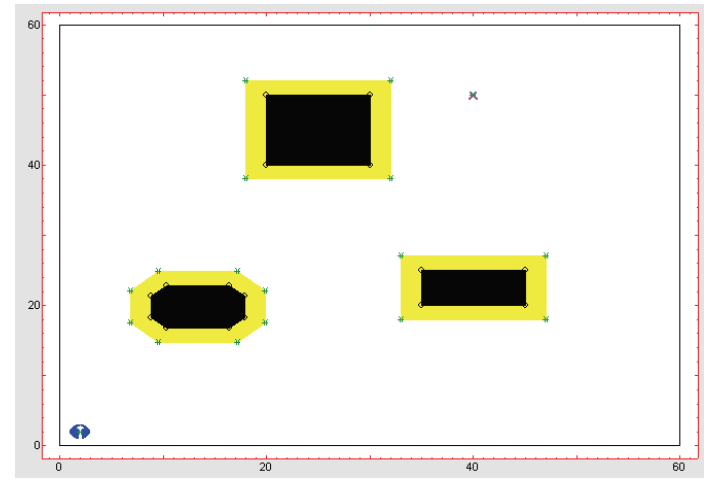

(a) Step 1. All visible points.

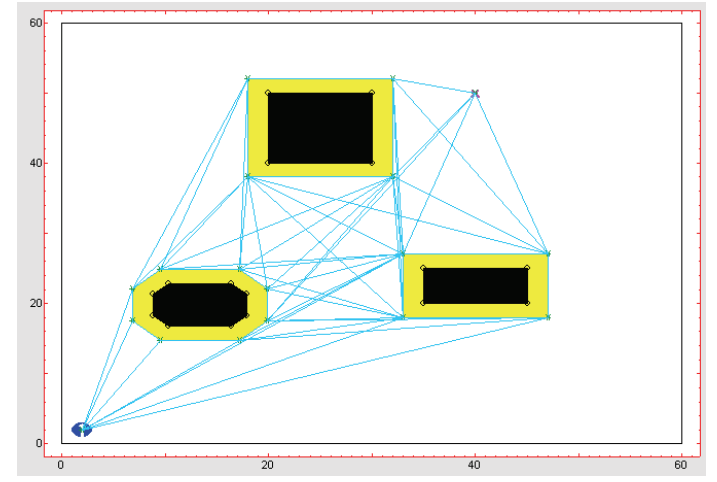

(b) Step 2. All possible paths.

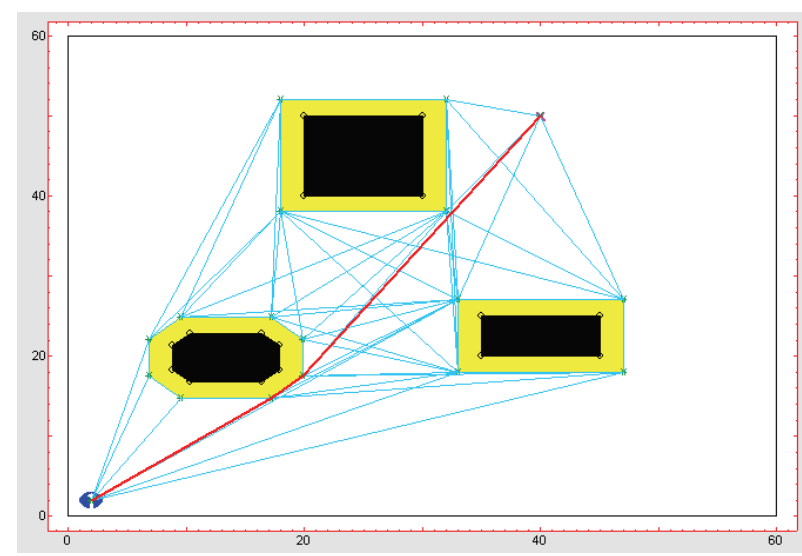

(c) Step 3. Minimum length path (Dijkstra).

Fig. 4. Visibility graph algorithm.

- Generalized Voronoi diagram GVD. A Voronoi diagram is formed by partitioning a plane into polygonal regions, each of which is associated with a given point (locus of points closer to that point than to any other given point). The edge separating two regions is composed of equidistant points 


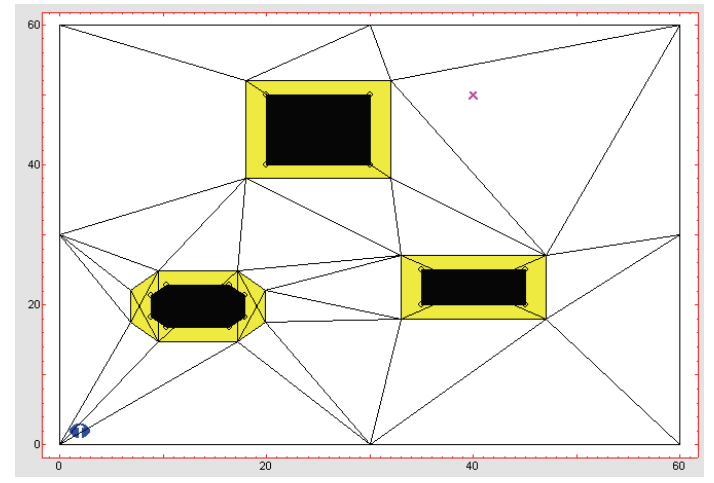

(a) Step 1. Delaunay triangulation.

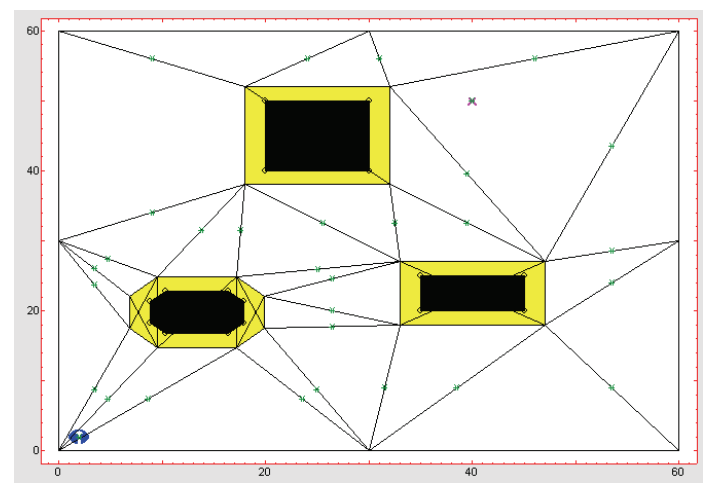

(b) Step 2. All possible visible points.

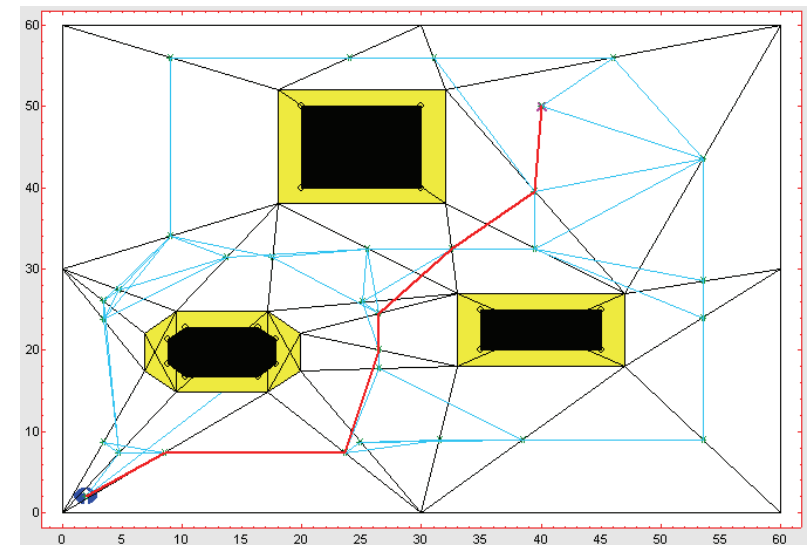

(c) Step 3. Minimum length path (Dijkstra).

Fig. 5. Approximated Voronoi algorithm.

from the given ones. When this diagram is used in robotics, an alternative Voronoi diagram (GVD) is generated, meaning the locus of equidistant points to obstacles and limits of the free space. The final set of points obtained define all possible paths to reach the goal point. A modified version of this algorithm has been implemented in the interactive tool by interactivity reasons, as shown in Fig. 5. First, the Delaunay triangulation is performed removing the triangles located inside of the objects [6]. Afterwards, the middle points of the triangle edges are obtained, these points being used in order to calculate all possible paths.

- Cell decomposition and quad-trees. These methods are based on dividing the free space or the composite space into regular or irregular cells. The possible paths will form a graph that joins selected points (such as the center of gravity) of all consecutive cells free of obstacles (see Fig. 6).

- WaveFront. In this algorithm the environment is represented by a two dimensional map of exact cell decomposition, each of its cells representing either a free-space or an obstacle [2], [21]. The distance cost for each cell is derived based on propagating the distance costs of the surrounding cells. The cell representing the destination point is given a distance cost equal to a small initial value (for instance, the value 1 or 2 in the tool). All other 


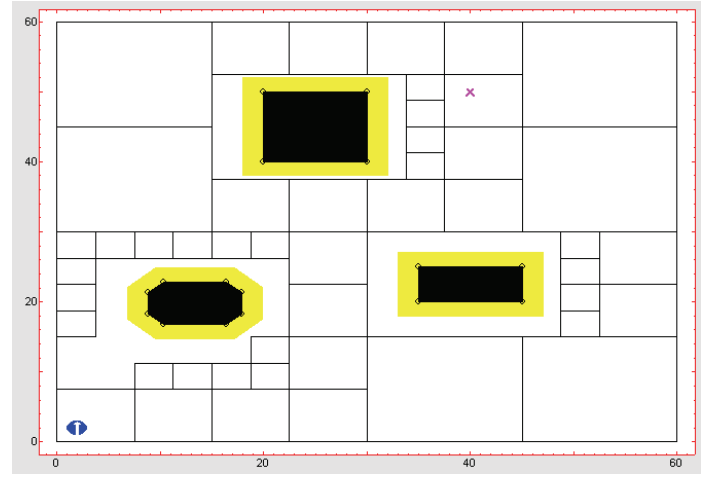

(a) Step 1. Cell decomposition.

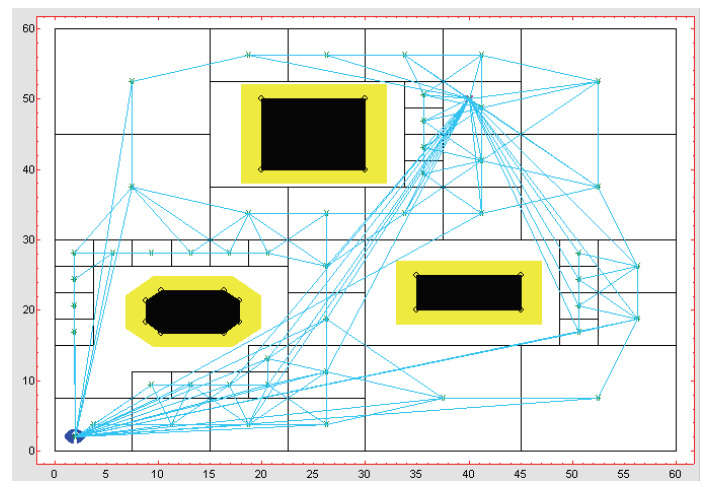

(c) Step 3. All possible paths.

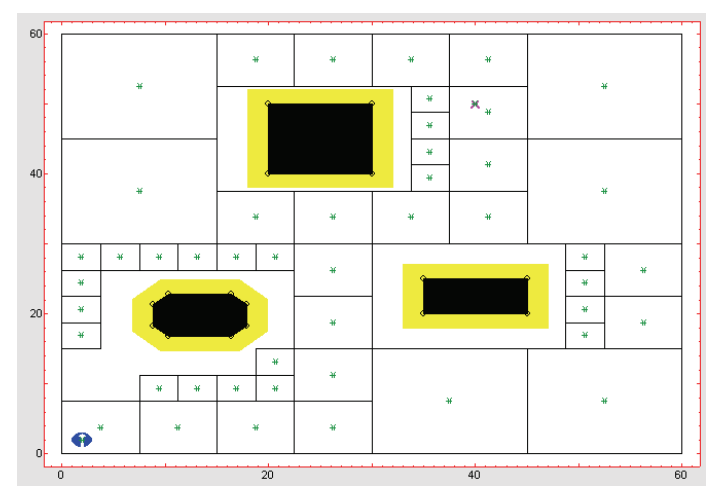

(b) Step 2. Central points.

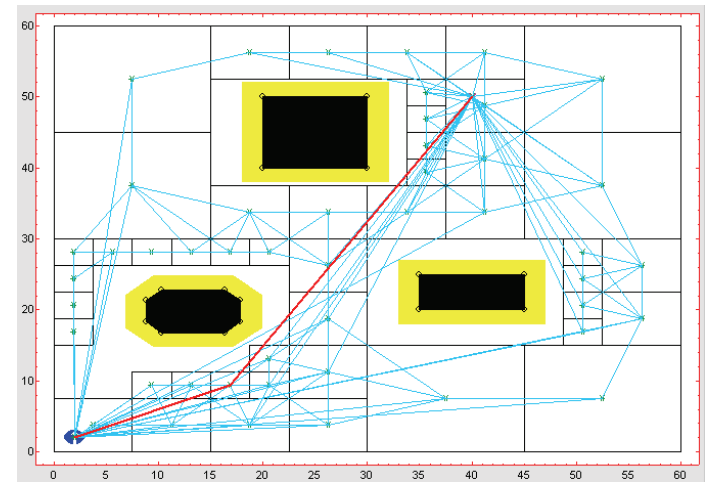

(d) Step 4. Minimum length path (Dijkstra).

Fig. 6. Cell decomposition algorithm.

cells are initially set to a very high value. The process is constructed as a two pass traversal of the 2D map array [21]: a forward raster order pass (left to right, top to bottom), and a reverse raster order pass (right to left, bottom to top). In each pass, each free-space cell (obstacles are skipped) is assigned a value of one greater than the least value of the four neighbors previously visited on that pass, this assignment occurs only if the new value is less than the previous value at that cell. Both steps are repeated until no further changes to cell assignment occur. The final distance cost at each cell represents the minimum number of cell steps to the destination's cell. The optimal path can be found from any starting point by looking at its 8-neighbor cells and moving to the one with the lowest distance cost. This process is repeated until the target cell is reached (see Fig. 7).

Once the possible paths have been computed, the next step consists in calculating the optimum path according to a certain criteria: minimum distance, minimum curvature, etc. The criterion selected in the tool has been the minimum distance. There is a wide variety of problems that go under the name "Shortest Path Problem". A determined algorithm must be selected based on the problem structure, the assumptions made, the type of the data, and the nature of the solutions desired. In the context of the path planning for mobile 


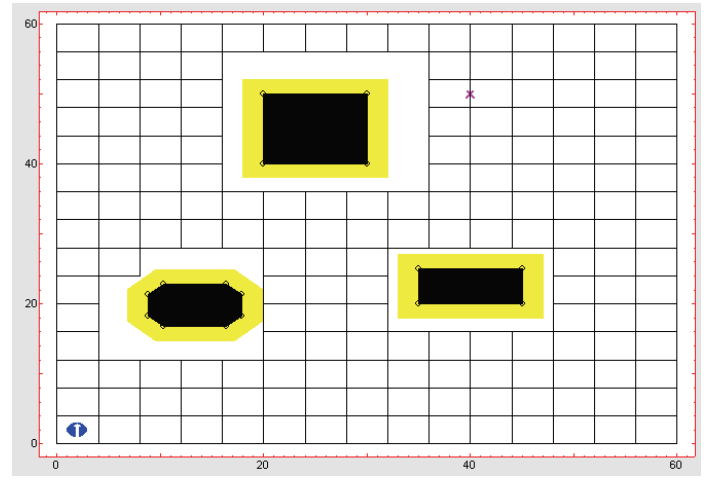

(a) Step 1. Cell decomposition.

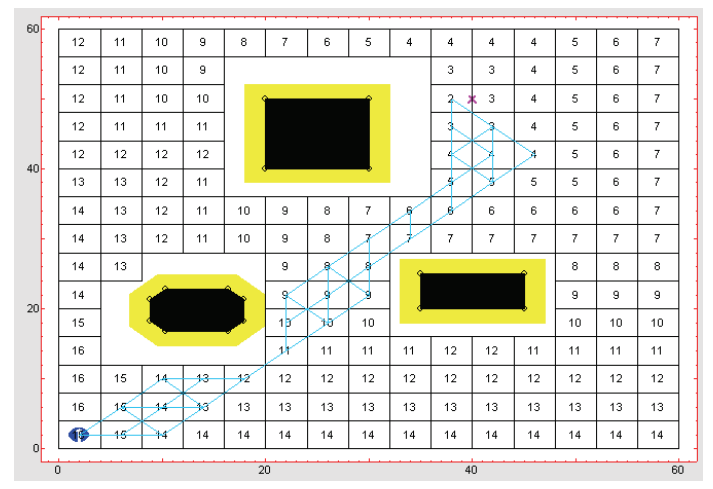

(c) Step 3. All possible paths.

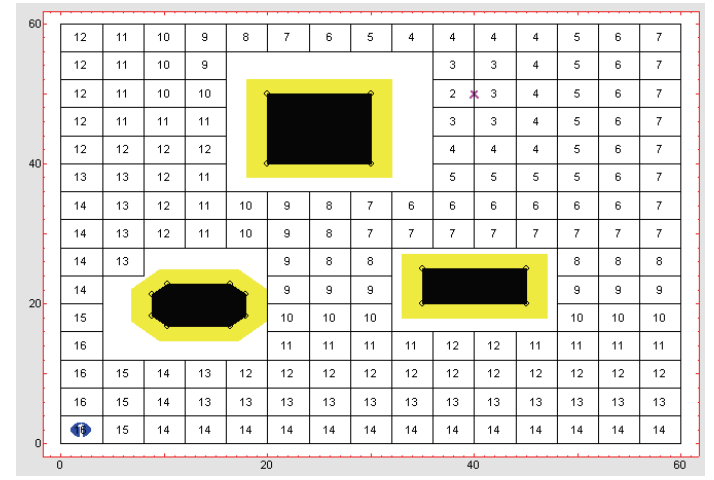

(b) Step 2. Logical values for cells.

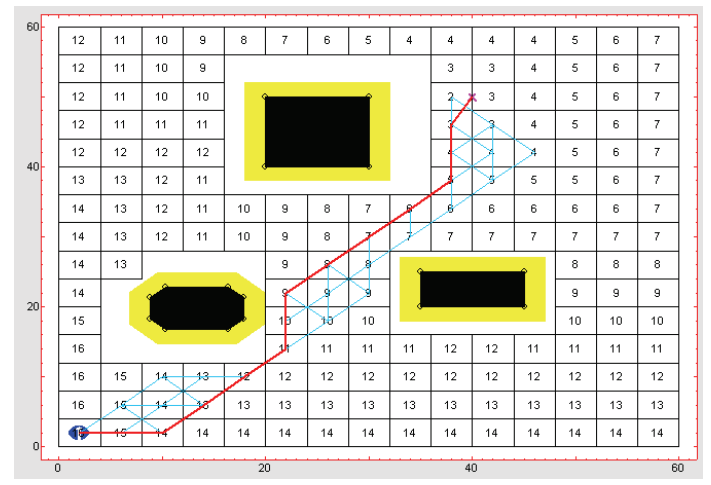

(d) Step 4. Minimum length path.

Fig. 7. WaveFront algorithm.

robots, the shortest path problem is defined as the problem of finding in a direct weighted graph the path with lowest cost from a source vertex to a set of target vertex.

This problem is a classic topic in the field of Combinatorial Optimization and in the area of Artificial Intelligence. In Combinatorial Optimization the Dijkstra algorithm in case of nonnegative arc distances, and the Modified Dijkstra algorithm in case of arbitrary distances, are well known. On the other hand, in Artificial Intelligence, this problem arises in the context of heuristic search based on a heuristic estimation function, which returns for each vertex an estimate of the length of the shortest path to the single target vertex, being A* algorithm the best known [30].

In this tool the Dijkstra algorithm has been used. It is necessary to indicate that the main objective of this tool is the study of the Robot Motion Planning, so the used method to obtain the shortest path in a graph does not influence to this objective. Furthermore, the computer engineering students have studied these algorithms in other subjects like "data structures".

After obtaining the shortest path, the next step is to calculate the control signals required to follow the optimum path. This task has been implemented 
using the pure pursuit method due to its simplicity and easy understanding by the students, although other strategies such as constrained predictive control [11] could be also used for these purposes.

\subsubsection{Pure Pursuit}

The pure pursuit is a geometric method which main assumption is that the curvature of the robot is constant during each sample time, in such a way that the robot follows the arc of a circumference between sampling instants. The movement of the robot centre is determined by its linear $(v)$ and angular $(\omega)$ velocities $(v=R$, where $R$ is the curvature radius and $\gamma=1 / R$ is the curvature). If an objective point on the trajectory is defined by its global coordinates $\left(x_{o b}, y_{o b}\right)$, the curvature required to reach this point (Fig. 8) is given by $[26]$ :

$$
\gamma=\frac{1}{R}=-2 \frac{\Delta x}{L^{2}}
$$

where $L$ is the distance to that goal point. It corresponds to a control law proportional to the lateral error $\Delta x$ to the goal point (Fig. 8), $\Delta x_{L}$ is defined in the robot local coordinates system. The proportional constant varies based on the inverse of the square of $L$. Once the desired curvature has been obtained, the next step will be to obtain the control signals to the actuators depending on the robot configuration treated in the next section. As the trajectory coordinates and the goal point in the trajectory $\left(x_{o b}, y_{o b}\right)$ are defined using a global reference system, it is necessary to take into account the robot orientation $\phi$ to obtain $\Delta x$ :

$$
\Delta x=\left(x_{o b}-x\right) \cos \phi+\left(y_{o b}-y\right) \sin \phi, \quad L=\sqrt{\left(x_{o b}-x\right)^{2}+\left(y_{o b}-y\right)^{2}}
$$

A practical method to apply the control law [26], is to determine at each sampling time the global coordinates of the robot $(x, y)$, to obtain the nearest point to the robot belonging to the trajectory $\left(x_{o b m}, y_{o b m}\right)$ and to select as next objective point in the trajectory $\left(x_{o b}, y_{o b}\right)$ that placed at a distance $d$ on the path from $\left(x_{o b m}, y_{o b m}\right)$. Then $L$ is calculated and the control law (1) can be applied.

\section{Local algorithms}

Local algorithms have as objective to guide the robot through an unknown environment using only the information provided by its on board sensors. 


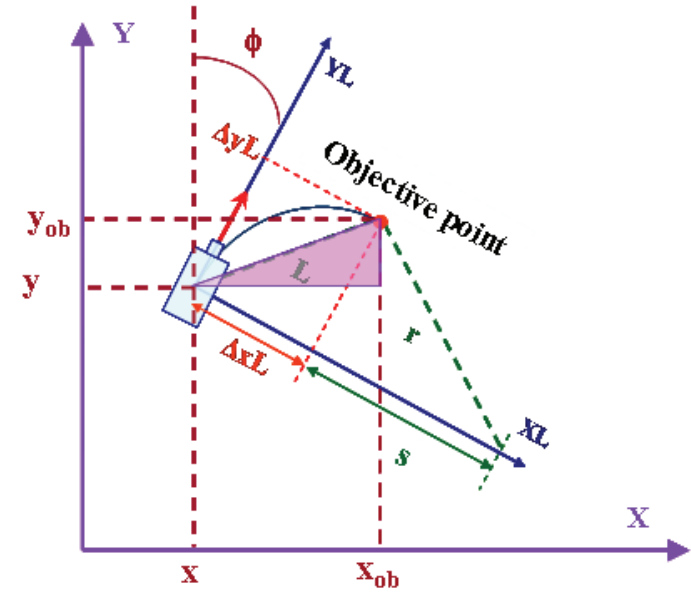

(a) Control law application.

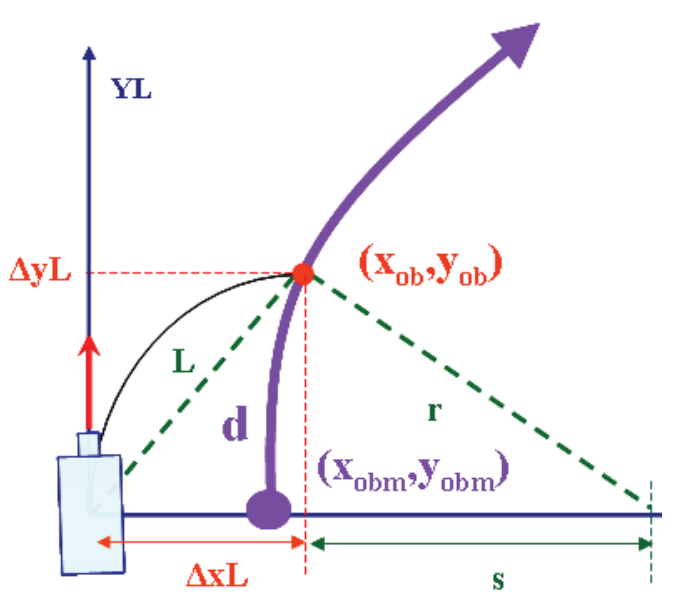

(b) Calculating the new objective point.

Fig. 8. Pure pursuit method.

The main advantages against global algorithms are that the required computational power is much smaller and are easier to implement. The main drawback is that in general, the solution (reaching the goal point) cannot be guaranteed, even if this exists (only some types of methods like BUG ones can provide some figures regarding convergence). The following local algorithms have been considered in the tool [2], [17], [20] (see Table 1):

- Potential function. It is based on the idea that the robot moves within a "field of forces". The goal point acts as an attraction pole while the obstacles are repulsion surfaces for the robot. A composition of attraction and rejection forces is performed to obtain the direction of the displacement all the time (see Fig. 9(a)):

$$
\begin{aligned}
\vec{F}_{\text {nav }} & =\vec{F}_{a t r}+\vec{F}_{\text {rep }} \\
\vec{F}_{\text {atr }} & =\frac{K_{a t r}\left(x_{t}-x\right)}{d_{t}} \vec{i}+\frac{K_{a t r}\left(y_{t}-y\right)}{d_{t}} \vec{j} \\
\vec{F}_{\text {rep }} & =\sum_{n} \vec{s}_{i} \\
\vec{s}_{i} & =-\left(C_{\text {rep }} \cos \left(\theta_{i}\right) \vec{i}+C_{\text {rep }} \sin \theta_{i} \vec{j}\right)
\end{aligned}
$$

where $\vec{F}_{\text {atr }}$ and $\vec{F}_{\text {rep }}$ are the attraction and repulsion forces respectively, $\left(x_{t}, y_{t}\right)$ is the target point, $(x, y)$ is the current point of the robot, $d_{t}$ the current distance to the target point, $\vec{s}_{i}$ is the repulsion force generated by each sensor $i$ detecting an obstacle, $\theta_{i}$ is the orientation of each sensor $i, n$ is the number of sensors, and $K_{a t r}$ and $C_{r e p}$ are the weighting factors for the attraction and repulsion forces respectively (tuning parameters).

- $B U G$. The robot is provided with contact sensors when using this method, and tries to reach the goal point straight forward. The robot surrounds the detected obstacles till reaching again the straight line joining the initial and goal points, following it till finding a new obstacle and so on (see Fig. 9(b)). 
- VISBUG. It is a modification to the BUG algorithm but using finite range distance sensors, in such a way that it is not necessary to contact with the obstacles. So, direction changes are performed in advance and non-convex zones of the obstacles are avoided (see Fig. 9(c)).

It is also possible to combine global and local algorithms: the first ones can be used to obtain a minimum length path within a known environment and once the path tracking has started, the local algorithms can be used to avoid collisions with unknown obstacles or to compensate for environment imperfections. This last option combining algorithms has not been implemented in the interactive tool in order to facilitate the understanding of the different algorithms separately.

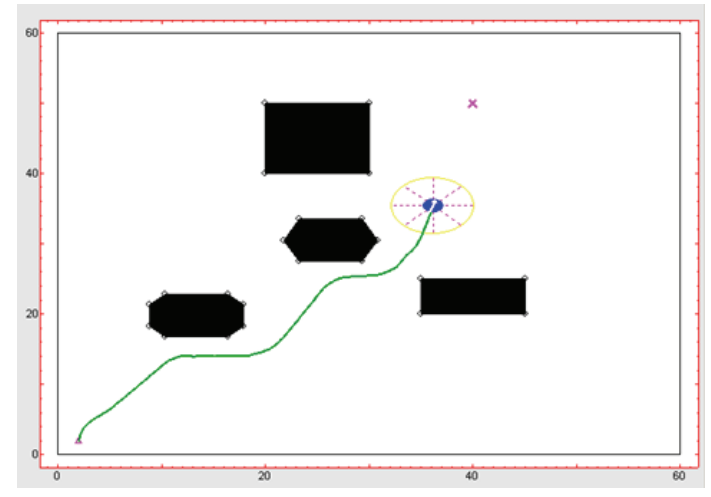

(a) Potential algorithm.

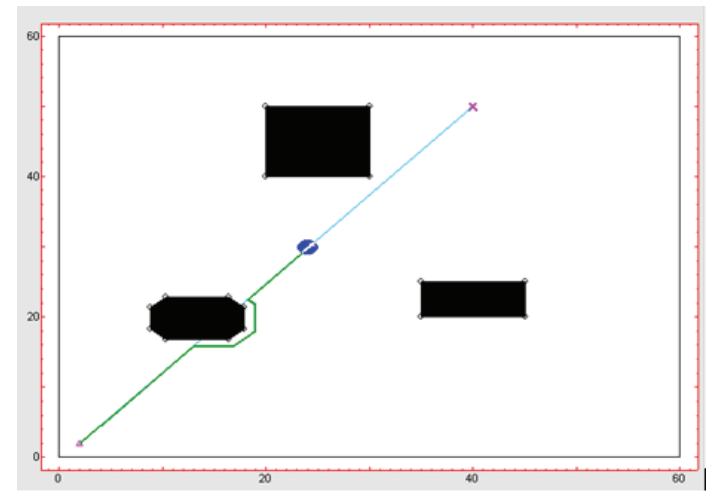

(b) $B U G$ algorithm.

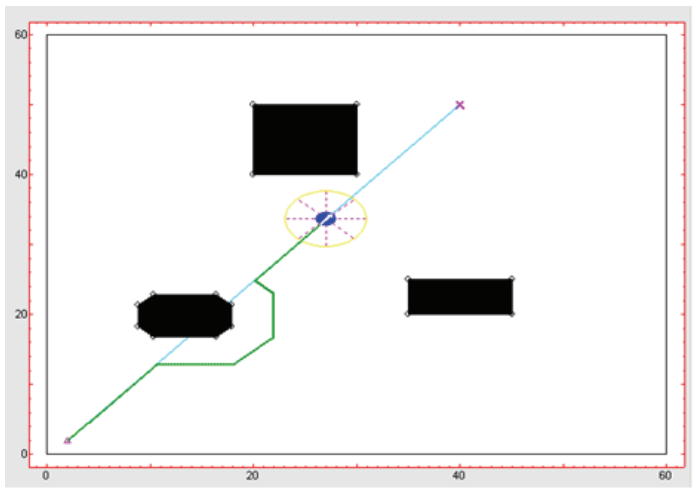

(c) VISBUG algorithm.

Fig. 9. Local algorithms.

\section{Robot kinematics}

The implementation of the path planning and tracking tasks requires the estimation of the robot position in relation to the same absolute reference system where the coordinates of the trajectories are defined. The kinematic models 


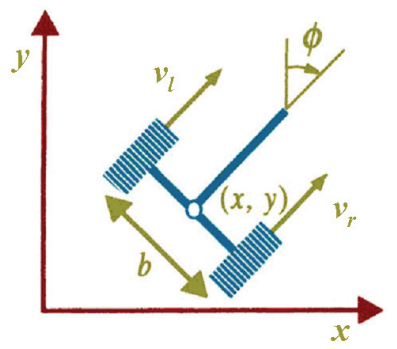

(a) Differential.

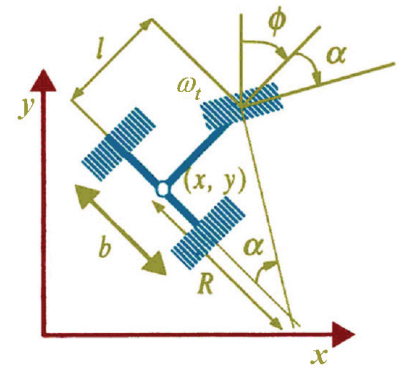

(b) Tricycle.

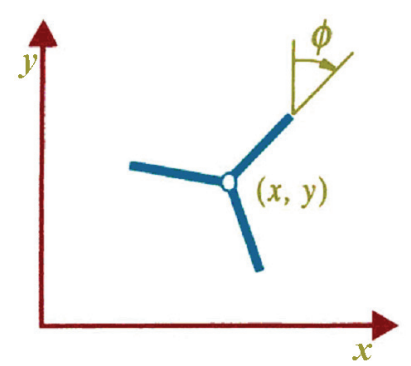

(c) Synchronous.

Fig. 10. Robot configurations.

of the robot are used to estimate the robot position $(x, y)$ and orientation $(\phi)$ all the time (at each sampling time when implementing digital control, with discrete-time versions of the kinematics, as done in this work). As has been pointed out in the previous section, in all the cases the robot displacement is determined by its linear $(v)$ and angular $(\omega)$ velocities in such a way that, if the linear velocity is fixed, knowing the desired curvature from the pure pursuit (or other) method $\omega$ can be determined and thus the robot displacement. The different kinematics are described as follows:

- Differential mechanism (Fig. 10(a)). The robot has two motored wheels, so that the control variables are the angular speed of each one $\left(\omega_{l}, \omega_{r}\right)$. The kinematics are described by discretized versions of (7)-(9):

$$
\begin{aligned}
x^{\prime} & =-\frac{r \sin \phi}{2} \omega_{l}-\frac{r \sin \phi}{2} \omega_{r} \\
y^{\prime} & =\frac{r \cos \phi}{2} \omega_{l}+\frac{r \cos \phi}{2} \omega_{r} \\
\phi^{\prime} & =-\frac{r}{b} \omega_{l}+\frac{r}{b} \omega_{r}
\end{aligned}
$$

where ' represents time derivative, $r$ is the wheel radius, and $b$ is the separation of the wheels. The relation with $v$ and $\omega$ is given by:

$$
\begin{aligned}
\omega_{l} & =\frac{v-(b / 2) \omega}{r} \\
\omega_{r} & =\frac{v+(b / 2) \omega}{r}
\end{aligned}
$$

- Tricycle/Ackermann (Fig. 10(b)). This kind of robots has three/four wheels: one/two in the front (motorized and directional wheels) and two wheels in the back. The kinematics are given by the discretization of

$$
\begin{aligned}
x^{\prime} & =-v \sin \phi \\
y^{\prime} & =v \cos \phi \\
\phi^{\prime} & =v \gamma
\end{aligned}
$$


The control signals in this configuration are the direction angle $\alpha$ and the angular velocity of the directional wheel $\omega_{t}$, given by:

$$
\begin{aligned}
\omega_{t} & =\frac{v_{t}}{r}=\frac{\sqrt{v^{2}+\omega^{2} l^{2}}}{r} \\
\gamma & =\frac{\tan \alpha}{l}
\end{aligned}
$$

where $l$ is the robot length and $r$ is the radius of the directional wheel.

- Synchronous (Fig. 10(c)). It contains transmissions to allow positioning all the wheels with an angular speed $\omega$ simultaneously, and moving the robot with a linear speed $v$. The kinematics is given by

$$
\begin{aligned}
x^{\prime} & =-v \sin \phi \\
y^{\prime} & =v \cos \phi \\
\phi^{\prime} & =\omega
\end{aligned}
$$

with the angular speed $\omega$ being the control signal.

\section{Illustrative examples}

Several examples are shown in this section to demonstrate the main capabilities of the tool, although as has been pointed out, its main characteristic - the interactivity - is difficult to be reflected in a written text. Nevertheless, some of the advantages of the application are shown below. The reader is cordially invited to visit the Web site [12] to experience the interactive features of the tool.

\subsection{Global algorithms}

The environment shown in Fig. 4-7 incorporates all the steps related to the application of global path planning algorithms (calculation of the convex hull, obstacles enlarging, determination of initial and goal points, and determination of all possible paths), as well as the minimum distance path calculation. The minimum length trajectory is obtained using the Dijkstra algorithm and it is shown in red color in the interactive tool. The tracking of this trajectory is performed using the pure pursuit method after selecting the desired robot kinematics and Run option from the Settings menu. Fig. 11 shows the results obtained using differential kinematics for all global algorithms for the same environment that in Fig. 4-7. Thus the tool allows studying the differences between the available global algorithms. From the previous results, the visibility graph algorithm provides the shortest and smoothest path, while the modified 
Voronoi algorithm the longest and most angular one. However, from a safety point of view (distance to obstacles), the Voronoi and wave front algorithms provide the best results.

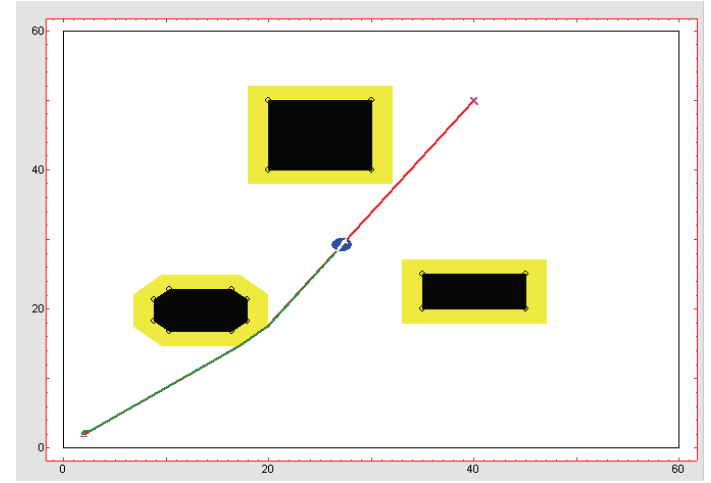

(a) Visibility graph.

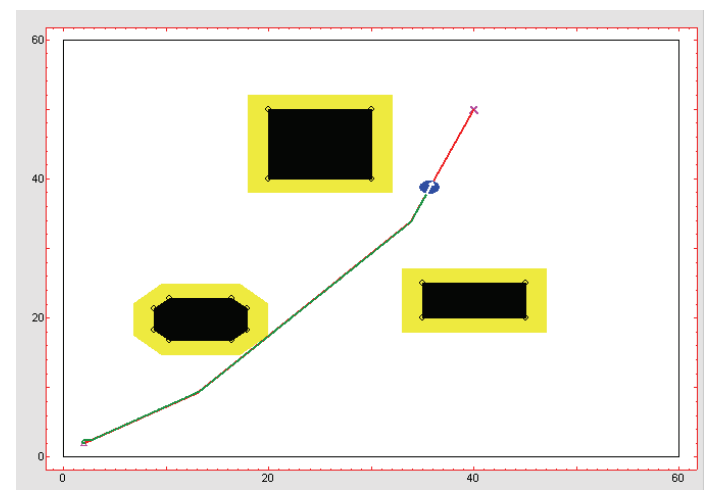

(c) Cell decomposition.

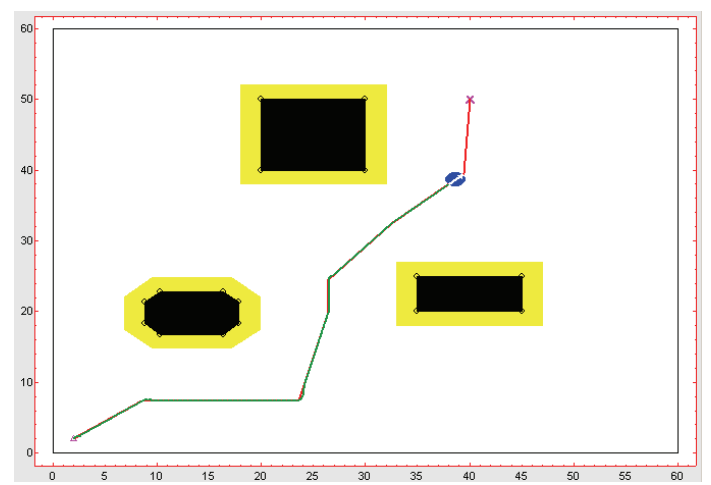

(b) Voronoi diagram.

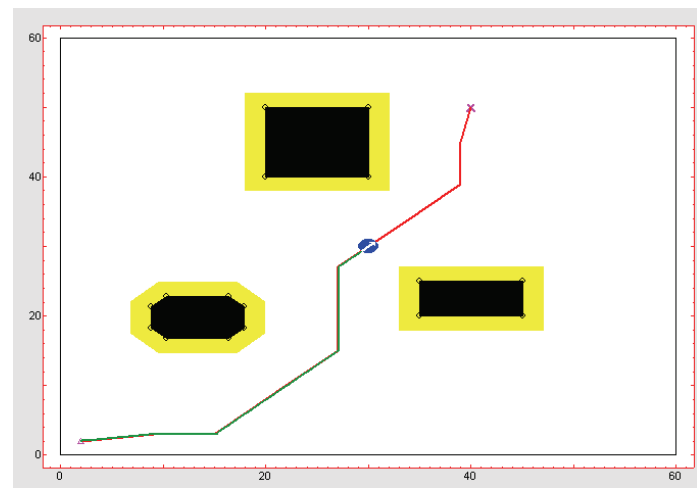

(d) WaveFront.

Fig. 11. Tracking examples for global algorithms.

\subsection{Obstacles insertion}

The tool allows modifying, including, and deleting objects in the environment. Once any of these actions is performed the used algorithm must take into account the new conditions to find the final point. In the case of global algorithms, it is necessary to calculate the new possible ways for determining if some of them are more optimal than the previous path obtained. When using local algorithms the changes are not so important since the robot has no knowledge of any modification in the environment. Fig. 12 shows the effect of environment modifications for the examples of Fig. 4, 7, and 9, for global and local algorithms. 


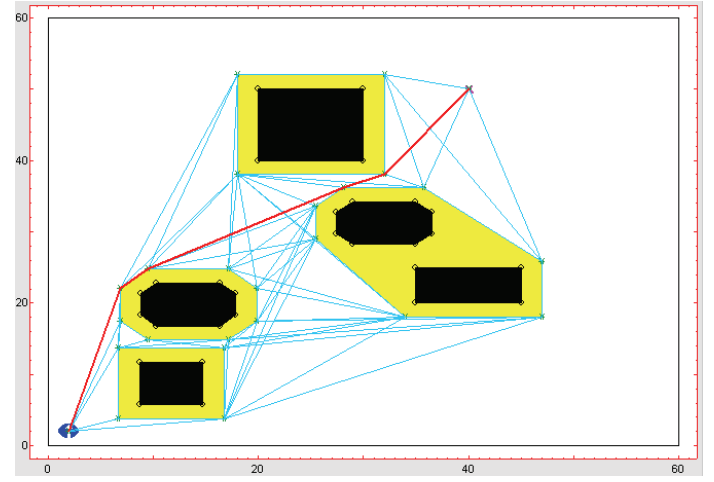

(a) Visibility graph.

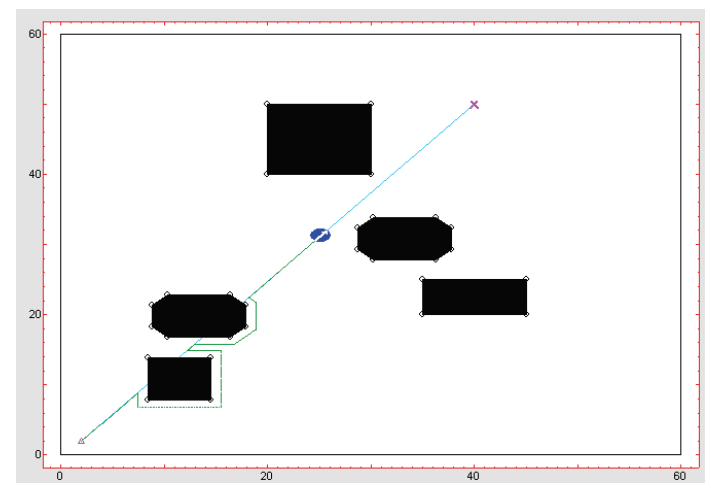

(c) BUG.

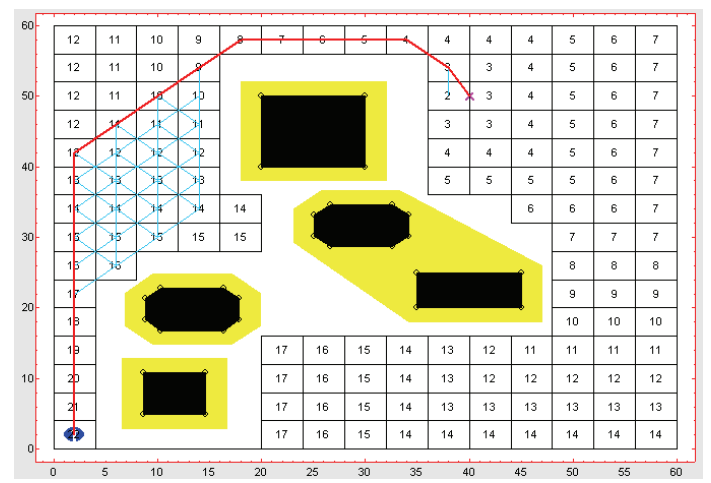

(b) WaveFront.

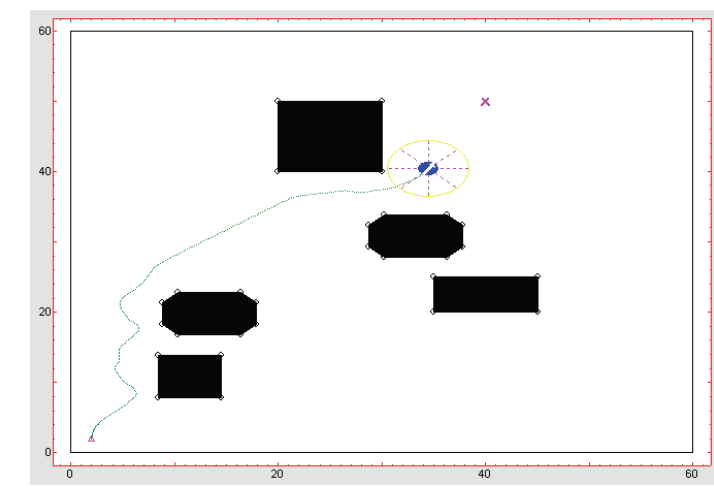

(d) Potential.

Fig. 12. Effect of environment modification in different algorithms.

\subsection{Constraints over the control signals and kinematics}

The wheels speed or the turn angle of the robot have often physical limitations. These constraints can be incorporated interactively in the graphs located at the bottom right of the tool. In the same way, using the interactive tool it is possible to compare the performance under different types of robot kinematics. For instance, Fig. 13 shows the three robot morphologies and the control actions associated to each kinematics. In the cases of tricycle and synchronous kinematics some physical limitations have been taken into account restricting $\alpha$ to $\pm 1 \mathrm{rad}$ and $\theta$ to $\pm 1,2 \mathrm{rad}$, respectively. From the results, it can be summarized that the differential mechanism kinematics is the only one that provides turn angle equal to zero. It can be also seen the bad behavior of the tricycle one due to its large turn angle (only one free wheel) and the constraints imposed during the simulation. 


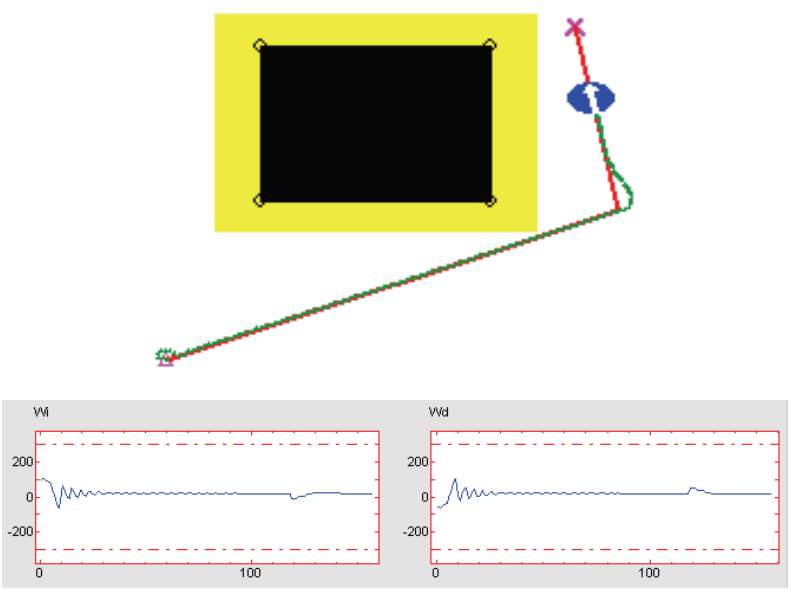

(a) Differential mechanism.

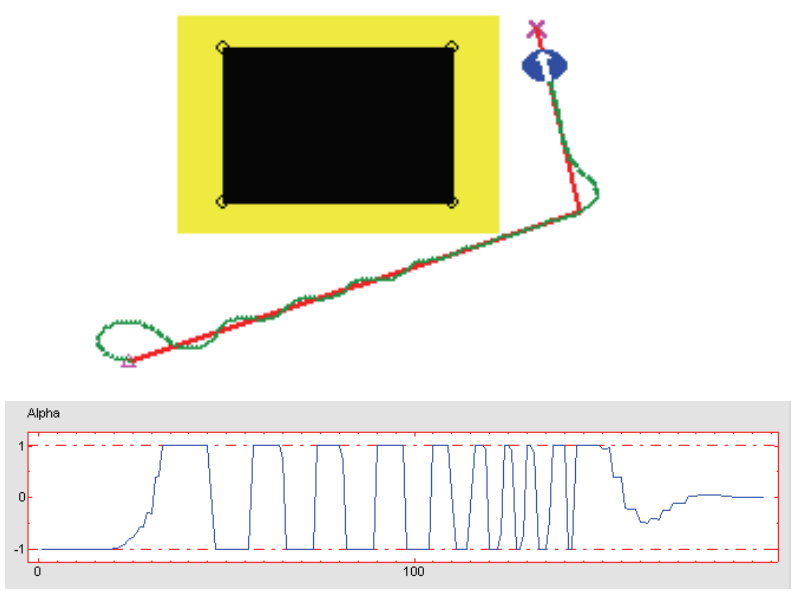

(b) Tricycle.
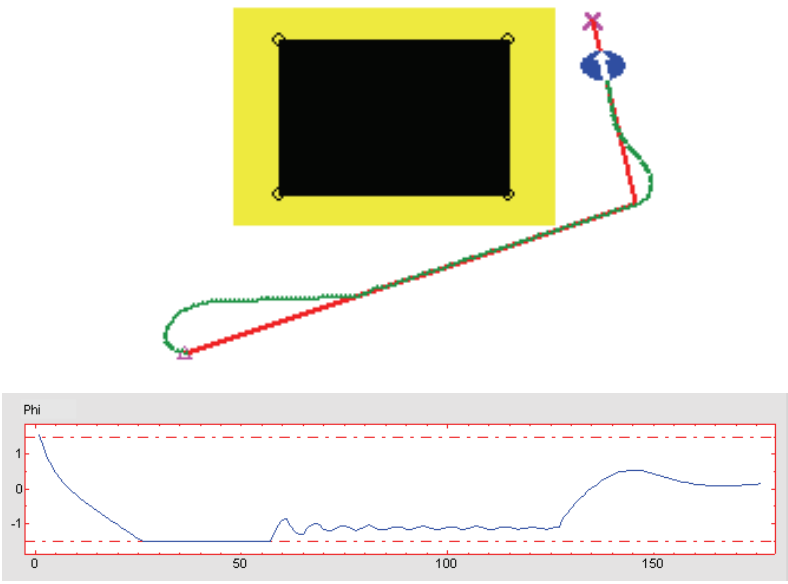

(c) Synchronous.

Fig. 13. Studying different kinematics.

\section{Teaching improvements using the interactive tool}

In our personal experience, the development of practical sessions is incrementally performed, from those involving different robot kinematics to those treating path tracking, in such a way that the development of one lab session requires to complete the previous ones.

Traditional teaching methods are used to explain the theory required to develop the lab sessions, with the help of the tool (used by the teachers during theoretical lectures). As it is well known, mobile robotics involves a strong mathematical content and thus graphical abstractions are commonly used to facilitate the understanding of the concepts. In this way, when explaining these concepts (as happens with general control theory), all the design steps are translated into a synthesis phase where the mathematical structure of the 
problem is determined and after, an analysis phase has to be carried out to analyze the results. This iterative process performed in two phases has been merged into one in the interactive tool.

The tool is also used by the students to analyze and compare the results with those of laboratory sessions. The implementation of the algorithms in these lab sessions is performed in Matlab/Simulink (alternatively in $\mathrm{C}++$ or Java) and the proposed lab sessions for a typical 60 hours course are (30 hours for lab sessions):

- Lab session 1. Learning and implementation of different robot kinematics: differential, tricycle, and synchronous. A discretized version of the algorithms is implemented, without incorporating dynamics.

- Lab session 2. The objective of this session is to implement and test the pure pursuit method using as robot simulator the kinematic model developed in the previous lab session.

- Lab session 3. The objective is the implementation of one global algorithm (visibility graph, GVD or quadtrees) and one local method (potential, BUG, VISBUG) selected by the lecturer.

- Lab session 4. The next step consists of building, programming (in C language and/or assembler) and controlling a TRITTON mini-robot (Fig. 14(a)) [36] or a PeopleBot robot. [37] (Fig. 14(b)). Students have to implement the pure pursuit method in this robots in order to track a path which representative points have been previously calculated using one of the algorithms tested in lab session 3 for a predefined environment.
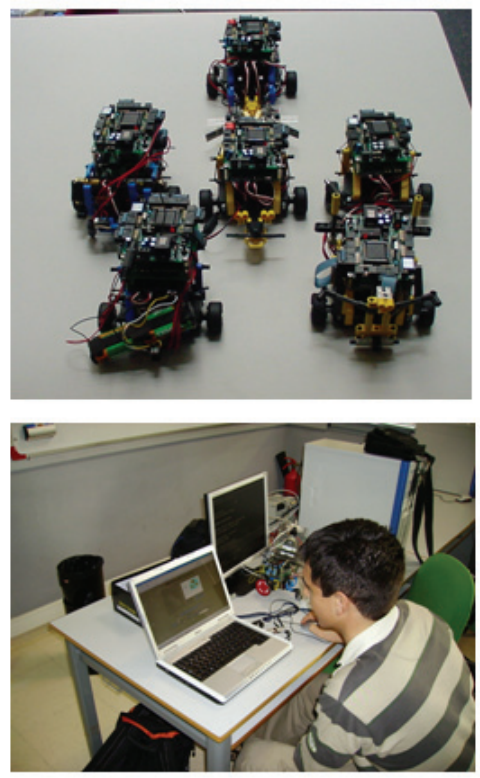

(a) Tritton.

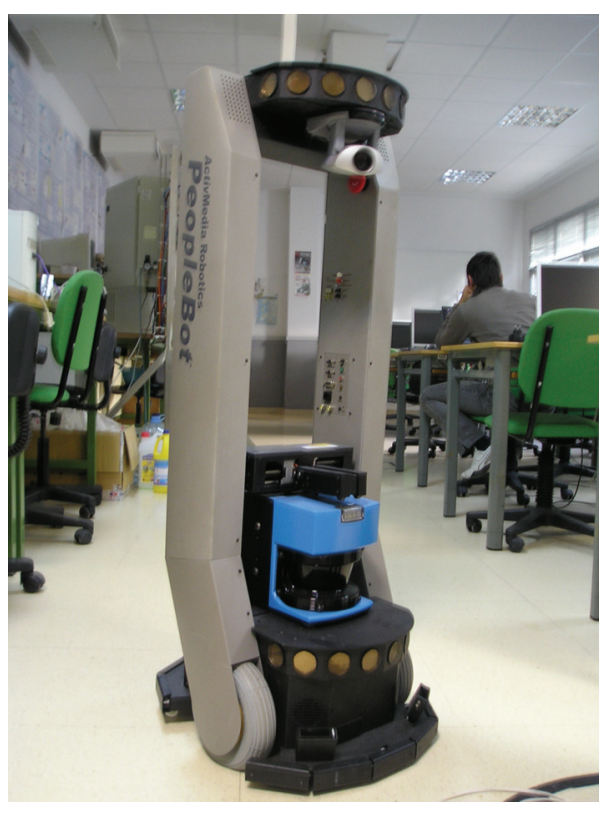

(b) Peoplebot.

Fig. 14. Studying with different mobile robots. 
From the point of view of theoretical sessions, the use of the tool helps to explain the performance and differences between different robot kinematics, how the constraints affect the performance, the effect of modifying different parameters, changes in the environment and navigation algorithms, etc. In the case of the lab session 4 the interactive tool offers the possibility of studying and simulating the robot behavior previous to testing it in the real environment. For instance, a typical environment in which the session is developed is composed of five obstacles of $8 \times 4 \mathrm{~cm}^{2}$ within a $1,2 \times 1,2 \mathrm{~m}^{2}$ square locus. Fig. 15 shows both a simulation with the interactive tool and a photo of the robot in the real environment.

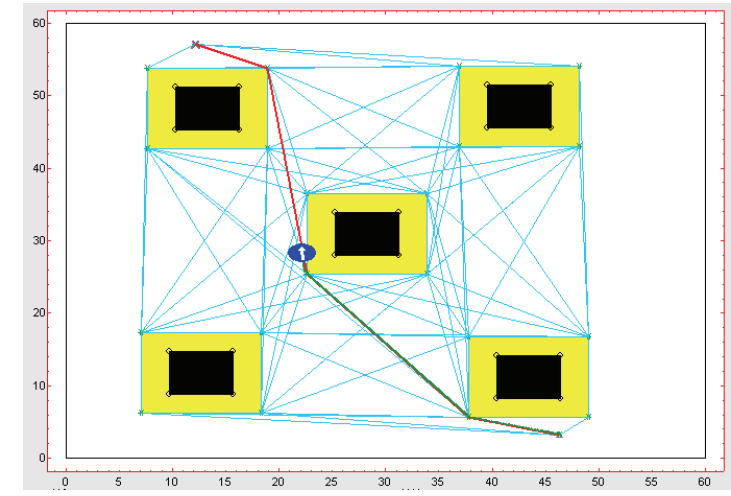

(a) Interactive Simulation.

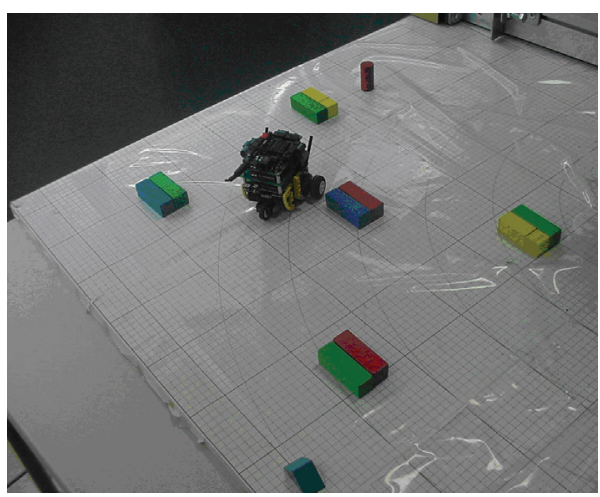

(b) Real test.

Fig. 15. Using the Interactive Tool previous to a real test.

\subsection{Criticism (and self-criticism) and feedback from the students}

The interactive tool then helps the students both to understand the concepts and also to test the algorithms in many different (and even atypical) conditions.

Regarding the feedback from the students, the teachers opened forums by raising diverse questions regarding the schedule of the course, contents, etc. The general opinion of the students (20 answers were obtained the fist year) is that the use of interactive tools was surprisingly positive for them, as they had the possibility of putting into practice the achieved knowledge. The main criticism in many cases was related to the computational burden associated to some of the algorithms (e.g. GVD), that produces a lost of realism in interactivity as the students should have to wait for a moment to see the effects of the performed changes instead of observing them as the manipulations are carried out. Anyway, the obtained results are very promising and a great effort is currently being performed to optimize the performance of the tool under all the considered conditions. 


\section{Conclusions and future work}

This work shows an interactive tool aimed at helping the students to understand the basis of mobile robot navigation and letting them modifying different characteristics such as robot kinematics, kind of path planning algorithm, the shape of the obstacles, etc. Typical robotics simulation tools are expensive and thus it is difficult to have many licenses for the students. The same applies to real robots. The use of interactive tools as that presented in this paper constitutes an alternative to classical teaching tools. The developed interactive tool is free and it can be obtained in English and in Spanish including also a couple of relevant tutorials [12]. It is available at http://www.calerga.com/ or http://aer.ual.es/mrit/ and it can run under Windows, Linux, and MAC operating systems.

Future works will include:

- Improving the computational efficiency of some of the implemented algorithms.

- The incorporation of new path tracking algorithms, such as constrained predictive control, as the authors have much experience in this kind of control techniques [11] and may explicitly take into account constraints acting on the control signals.

- Multivariable control for velocity and orientation.

- Inclusion of multiple robots.

- Development of a virtual lab by allowing its use through Internet and possible adaptation to a remote lab using in this case Sysquake Remote.

\section{Acknowledgments}

This work was supported by the Spanish CICYT and FEDER funds under grants DPI2004-07444-C04-04 and DPI2004-01804. The authors would like to thank Mr. Oliver Rodríguez López, Mr. Manuel Pasamontes, and Dr. Yves Piguet for their help and suggestions.

\section{References}

[1] S. Athanasiou, I. Poulakis, P. Tsanakas, N. Koziris, TALOS: An interactive Web-enabled educational environment on mobile robot technology, in: Proceedings of 10th Mediterranean Electrotechnical Conference I, Madrid (Spain), 2000, pp. 387-395. 
[2] H. Choset, K. M. Lynch, S. Hutchinson, G. Kantor, W. Burgard, L. Kavraki, Principles of Robot Motion. Theory, Algorithms, and Implementations, The MIT Press, 2005.

[3] C. Cosma, M. Confente, D. Botturi, P. Fiorini, Laboratory tools for tobotics and automation education, in: Proceedings of IEEE International Conference on Robotics and Automation, Tapei (Taiwian), vol. 3, 2003, pp. 3303-3308.

[4] F. Dai, Integrated planning of robotic applications in a graphic-interactive environment, Robotics and Autonomous Systems 8 (1991) 311-322.

[5] A. G. Demitriou, A. H. Lambert, Virtual environments for robotics education: an extensible object-orientation platform, IEEE Robotics and Automation 12 (4) (2006) 75-91.

[6] M. de Berg, M. van Kreveld, M. Overmars, O. Schwarzkoph, Computational geometry: algorithms and applications, Springer-Verlag, 2000.

[7] S. Dormido, Control learning: present and future, Annual Reviews in Control 28 (1) (2004) 115-136.

[8] P. Ehlert, The use of artificial intelligence in autonomous mobile robots, Research Report, Knowledge Based Systems Group, Delft University of Technology, 1999.

[9] A. Elnagar, L. Lulu, A global path planning Java-based system for autonomous mobile robots, Science of Computer Programming 53 (1) (2004) 107-122.

[10] A. Elnagar, L. Lulu, A visual tool for computer supported learning: The robot motion planning example, Computers and Education, in press (corrected proof available from 2005).

[11] J. L. Guzmán, M. Berenguel, S. Dormido, Interactive teaching of constrained generalized predictive control, IEEE Control Systems Magazine 25 (2) (2005) 79-85, available: http://aer.ual.es/siso-gpcit/.

[12] J. L. Guzmán, O. López, M. Berenguel, F. Rodríguez, S. Dormido, MRIT - Mobile Robotics Interactive Tool, UAL/UNED Internal Report, available: http://aer.ual.es/mrit/ and http://www.calerga.com, 2005.

[13] J. L. Guzmán, F. Rodríguez, M. Berenguel, S. Dormido, MRIT: Mobile robot interactive tool, in: Proceedings of the IFAC Workshop on Internet-Based Control Education IBCE04, Grenoble (France), 2004.

[14] J. L. Guzmán, Interactive control system design, Ph.D. Thesis, Deparment of Lenguajes y Computación, University of Almería, Spain, 2006.

[15] B. Heck (editor), Enhancing classical control education via interactive GUI design, IEEE Control System Magazine 19 (3) (1999) 35-58.

[16] J. Hrabec, Autonomous mobile robotics toolbox SIMROBOT, avilable: http://www.uamt.feec.vutbr.cz/robotics/, 2000. 
[17] M. Jenkin, G. Dudek, Computational principles of mobile robotics, Cambridge University Press, 2000.

[18] M. Johansson, M. Gäfvert, K. Åström, Interactive tools for education in automatic control, IEEE Control Systems Magazine 18 (3) (1998) 33-40.

[19] R. Kelly, S. Dormido, C. Monroy, E. Díaz, Learning control of robot manipulators by interactive simulation, Robotica 23 (2005) 515-520.

[20] J. C. Latombe, Robot motion planning, Kluwer Academic Publishers, 1991.

[21] M. Marouqi, R. Jarvis, Covert robotics: covert path planning in unknown environments, in: Proceedings of the Australasian Conference on Robotics and AutomationCanberra (Australia), 2003.

[22] The MathWorks Inc., Using Matlab. The language of technical computing, 2002.

[23] I. Maza, A. Ollero, HEMERO: A MATLAB-Simulink toolbox for robotics, in: Proceedings of the 1st Workshop on Robotics Education and Training, Weingarten (Germany), 2001.

[24] O. Michel, Khepera simulator version 2.0, available: http://diwww.epfl.ch/lami/team/michel/khep-sim/SIM2.tar.gz, 1999.

[25] U. Nehmzow, Mobile robotics, Springer, 2003.

[26] A. Ollero, Robótica. Manipuladores y robots móviles (in Spanish), Marcombo, 2001.

[27] T. Petrinic, E. Ivanjko, I. Petrovic, An interactive system for mobile robot navigation, in: Proceedings of the 5th International Workshop on Robot Motion and Control, Balatonfüred, Lake Balaton (Hungary), 2005.

[28] T. Petrinic, E. Ivanjko, I. Petrovic, AMORsim- A mobile robot simulator for Matlab, in: Proceedings of the 15th International Workshop on Robotics in Alpe-Adria-Danube Region, Balatonfüred, Lake Balaton (Hungary), 2006.

[29] Y. Piguet, SysQuake 3 user manual, Calerga Sarl, Lausanne (Switzerland), 2004.

[30] W. Pijls, A. Kolen, A general framework for shortest path algorithms, technical Report 92-08, University of Rotterdam (Holland), 2000.

[31] J. Sánchez, S. Dormido, F. Esquembre, The learning of control concepts using interactive tools, Computer Applications in Engineering Education 13 (1) (2005) 84-98.

[32] J. Sánchez, F. Morilla, S. Dormido, J. Aranda, P. Ruipérez, Virtual control lab using Java and Matlab: A qualitative approach, IEEE Control Systems Magazine 22 (2) (2002) 8-20.

[33] D. Srinivasagupta, B. Joseph, An Internet-mediated process control laboratory, IEEE Control System Magazine 23 (1) (2003) 11-18. 
[34] R. T. Vaughan, Stage: a multiple robot simulator, Technical Report IRIS00-394, Institute for Robotics and Intelligent Systems, University of Southern California, available: ftp://robot.usc.edu/pub/stage/stage_manual.ps.gz, 2000.

[35] B. Wittenmark, H. Hägglund, M. Johansson, Dynamic pictures and interactive learning, IEEE Control Systems Magazine 18 (3) (1998) 26-32.

[36] Microbótica S.L., available: http://www.microbotica.com/.

[37] Mobile robots company, available: http://www.activrobots.com/. 
Table 1

Parameters available in the interactive tool in order to configure the different navigation algorithms and the robot morphology.

\begin{tabular}{|c|c|}
\hline Parameter & Description \\
\hline $\operatorname{Tm}$ & Sample Time \\
\hline $\mathrm{d}$ & Distance to the objective point for the pure pursuit algorithm. \\
\hline Step number & Number of the steps to show for the selected algorithm. \\
\hline Quadtree area & Minimum area of the cells using the Quadtree global algorithm. \\
\hline 4-neighbours & Number of neighbours to use for the WaveFront algorithm. \\
\hline 8-neighbours & Number of neighbours to use for the WaveFront algorithm. \\
\hline Cell size & Minimum area of the cells using the WaveFront global algorithm. \\
\hline CounterClockWise & CounterClockWise direction for BUG and VISBUG algorithms. \\
\hline ClockWise & ClockWise direction for BUG and VISBUG algorithms. \\
\hline Sonar Range & Distance for the sonar sensors in the VISBUG and Potential algorithms. \\
\hline Attraction & Weighting factor in the Potential algorithm to weight the attraction of the target point. \\
\hline Repulsion & Weighting factor in the Potential algorithm to weight the repulsion of the obstacles. \\
\hline Orientation & Initial orientation of the robot. \\
\hline Speed & Speed of the robot. \\
\hline Size & Diameter of the robot. \\
\hline Radius & Radius of robot's wheels. \\
\hline Differential & Differential morphology of the robot. \\
\hline Tricycle & Tricycle morphology of the robot. \\
\hline Synchronous & Synchronous morphology of the robot. \\
\hline
\end{tabular}




\section{Short Authors' biography}

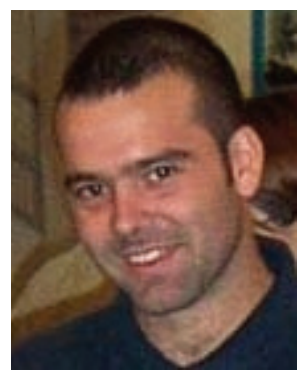

José Luis Guzmán obtained a computer science engineering degree in 2002 and a Ph.D. in 2006 both from the University of Almería, Spain, where he is an assistant professor and a researcher of the Automatic control, Electronics, and Robotics group. Currently his scientific interests are focus on the field of control education and robust model predictive control techniques, with applications to interactive tools, virtual and remote labs, and agricultural processes. Corresponding author. Address: Universidad de Almería, Dpto. de Lenguajes y Computación, Ctra. Sacramento s/n, 04120, Almería, Spain. Telephone number: +34 950015849. Fax number: +34 950015129. Email: joguzman@ual.es.

Manuel Berenguel is a Professor at the University of

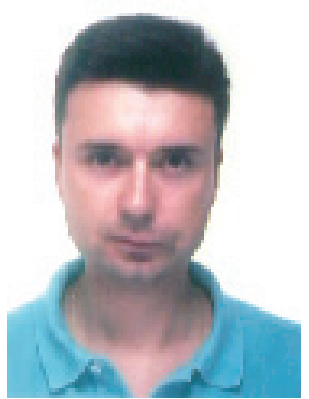
Almería, Spain. He obtained an industrial engineering degree and Ph.D. from University of Seville, Spain, where he received an award for the best engineering thesis of the year, and was also a researcher and associate professor for six years. His research interests are in the fields of predictive, adaptive, and robust control, with applications to solar energy systems, agriculture, and biotechnology. He has authored and co-authored more than 50 technical papers in international journals and conferences and is co-author of the book Advanced Control of Solar Plants, published by Springer in 1997.

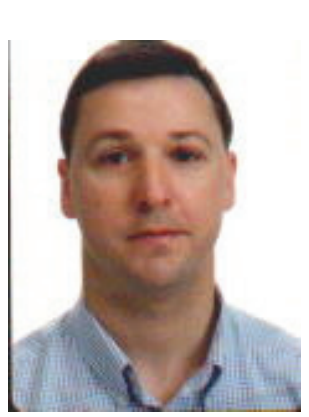

Francisco Rodríguez is an associate professor of systems engineering and automatic control at the University of Almera (Spain). He obtained his telecommunication engineering degree from Madrid Polytechnics University (Spain) and his Ph.D. degree from the University of Almera, in 2002. Now he is a researcher and member of the Automatic Control, Electronics, and Robotics group of the University of Almera. His scientific interests are focused to the application of modelling, automatic control, and robotics techniques to agricultural systems and education. 


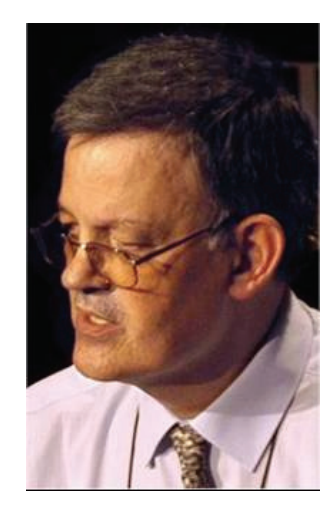

Sebastián Dormido holds a degree in physics from the Complutense University in Madrid, Spain (1968) and a Ph.D. from University of The Basque Country, Spain (1971). In 1981 he was appointed professor of control engineering at the Universidad Nacional de Educación a Distancia. His scientific activities include computer control of industrial processes, model-based predictive control, robust control, and model and simulation of continuous processes. He has authored and co-authored more than 150 technical papers in international journals and conferences. Since 2002 to 2006 he has been President of the Spanish Association of Automatic Control CEAIFAC. 